\title{
"Jissoku-Yochizu (1821)" which should be newly found as the small scale map of Japan originally surveyed by Inoh Tadataka
}

\author{
SUZUKI Junko, HOSHINO Yoshihisa, ARAI Keita, OTA Hiroshi * , KAWAMURA Hirotada, \\ SATO Wataru, SHINOHARA, Keiichi \\ mapotagis@gmail.com \\ * Corresponding author
}

Keywords: Jissokuyochizu, Old modern map Japan, Inoh Tadataka ,1821

\section{Abstract:}

Ino Tadataka: other cartographic findings of his surveying works have been found.With the confirmation of the existence of the second rare "Inozu Sho-zu" in Japan another great contribution is made to the knowledge of Ino Tadataka's work.

The works found are similar in color and character to the "Map of the coast of Japan (small paper)" preserved by the Tokyo National Museum, and both are described as “實測輿地圖” “Jissokuyochizu”. It is very likely that these two sheets were created around the same time.

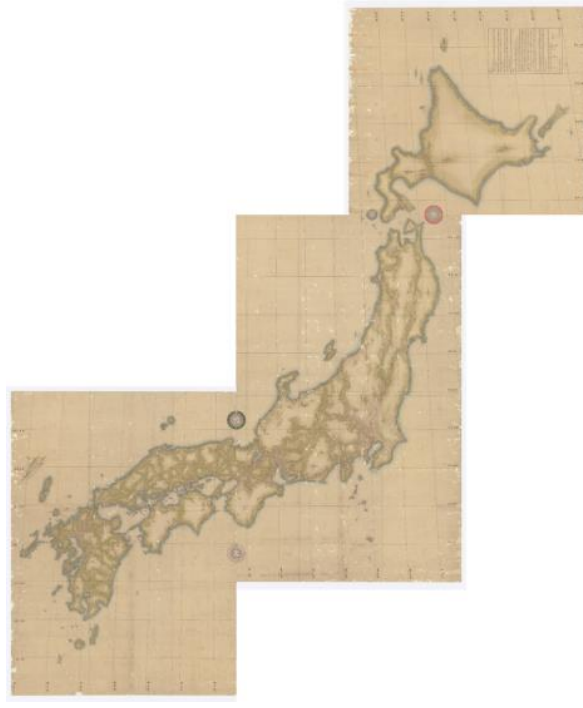

"Jissoku-Yochizu" small map

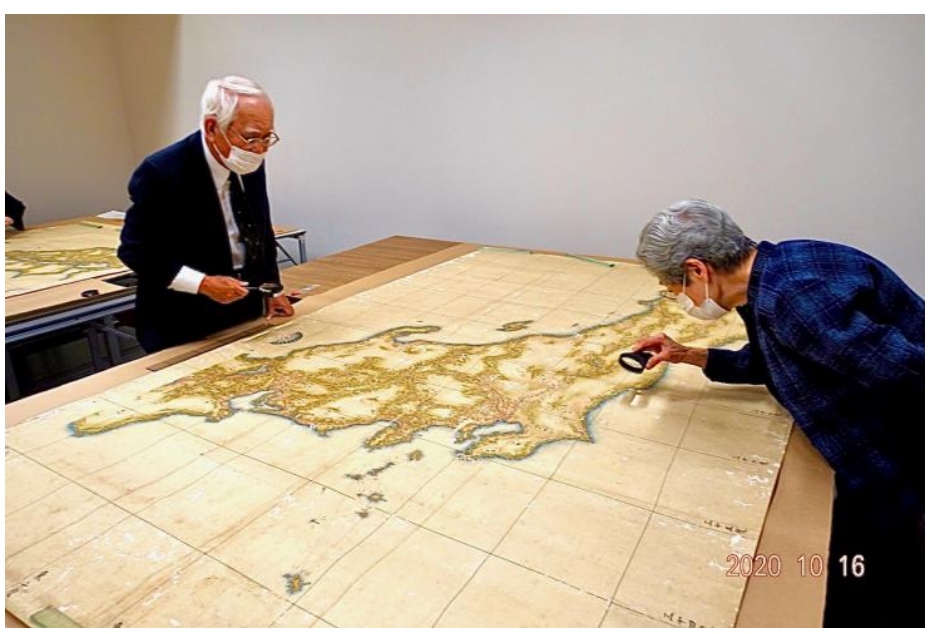

SUZUKI Junko, HOSHINO Yoshihisa

The Subcommittee of the Historical Material of Cartography and the Archive of maps, the Japanese Cartographic Society recently confirmed the second example of a small map (a complete set of three sheets) with a scale of $1 / 432,000$ created by the Ino Tadataka Survey Team.

The Japan map created by the Ino Tadataka Survey Team includes a large map at the scale of 1: 36,000, a medium map at the scale of 1: 1,216,000, and a small map at the scale of 1:432,000. The small maps are kept at the Tokyo National Museum The "Coastal map of Japan (small map)" consists of three maps of Hokkaido, Eastern Japan and Western Japan, owned by the museum. They have been recognized as the only ones created by the Ino Tadataka Survey Team.

All of this material was donated to the Zenrin Museum by a volunteer and, although its history is unclear, it does have a hole in the overlap which indicates that it was created by the Ino Tadataka Survey Team and is owned by the National 
Museum of Tokyo. Similar in color and font to the coast map (small map), it is very likely that both were created almost at the same time. This development map contains a legend that is not included in the "Japan Coastal Map (Small Map)" in the Tokyo National Museum collection.

Considering the aspect of the "Complete map of the coast of Japan" and proceeding with the cartographic research on the survey of Ino Tadataka, the small map confirms that it is the "Map of the coast of Japan (small map)" preserved. 